\title{
Tissue factor pathway inhibitor-2 inhibits the growth and invasion of hepatocellular carcinoma cells and is inactivated in human hepatocellular carcinoma
}

\author{
YONG XU ${ }^{1 *}$, XIAOLIN QIN ${ }^{2 *}$, JINJING ZHOU $^{2}$, ZHIGUANG TU $^{2}, \mathrm{XIAO} \mathrm{BI}^{3}$, \\ WUXIAN LI ${ }^{2}$, XIAOQING FAN ${ }^{2}$ and YI ZHANG ${ }^{3}$ \\ ${ }^{1}$ Pingshan People's Hospital, Shenzhen, Guangdong 518118; ${ }^{2}$ Key Laboratory of Laboratory Medical Diagnostics, \\ Ministry of Education, Chongqing Medical University, Chongqing 400016; ${ }^{3}$ The Second Clinical Medical College, \\ Jinan University, Shenzhen People's Hospital, Guangdong 518118, P.R. China
}

Received December 9, 2010; Accepted May 11, 2011

DOI: $10.3892 / \mathrm{ol} .2011 .340$

\begin{abstract}
Human tissue factor pathway inhibitor-2 (TFPI-2) is an extracellular matrix-associated Kunitz-type serine proteinase inhibitor that inhibits the plasmin- and trypsinmediated activation of matrix metalloproteinases and inhibits tumor progression, invasion and metastasis. Previous studies have shown that TFPI- 2 is downregulated in the progression of various tumors. The purpose of this study was to investigate the expression and function of TFPI-2 in hepatocellular carcinoma (HCC). In situ hybridization was used to detect human TFPI-2 mRNA and immunohistochemistry was performed to examine the role of TFPI-2 expression in hepatocarcinoma tissues. Cell proliferation was assessed using MTT assay. In situ hybridization and immunohistochemical analyses revealed that the expression of TFPI-2 in hepatocarcinoma tissues was markedly lower than that in tumor-adjacent normal hepatic tissues. Restored expression of TFPI-2 in $\mathrm{HepG}_{2}$ cells inhibits cell proliferation and invasion. Taken together, the results suggest that TFPI-2 has a tumor-suppression action and its inactivation may contribute to HCC.
\end{abstract}

\section{Introduction}

Hepatocellular carcinoma (HCC) is one of the most common primary tumors worldwide (1), and is particularly prevalent in China. It is a major complication of liver cirrhosis. HCC is characterized by a high rate of invasion and metastasis (2-4). Tumor invasion depends on extracellular matrix (ECM)-degrading

Correspondence to: Dr Yong Xu, Pingshan People's Hospital, 19 Renmin Road, Pingshan District, Shenzhen, Guangdong 518118, P.R. China

E-mail: xuyong_2000@tom.com

${ }^{*}$ Contributed equally

Key words: human tissue factor pathway inhibitor-2, hepatocarcinoma, $\mathrm{HepG}_{2}$, proliferation, invasion proteases, particularly matrix metalloproteinases (MMPs) in the tumor microenvironment $(5,6)$. Particularly in highly aggressive malignant tumors, MMPs are overexpressed in tumor and/or stromal cells and secreted into the ECM (7-9).

Tissue factor pathway inhibitor-2 (TFPI-2) is a $32-\mathrm{kDa}$ serine protease inhibitor comprising three kunitz-type domains homologous to TFPI, also known as placental protein 5 (PP-5) and matrix serine protease inhibitor (MSPI) $(10,11)$. The gene that encodes for TFPI-2 has been mapped to chromosome $7 \mathrm{q} 22$ by fluorescence in situ hybridization (12). TFPI-2 is abundantly expressed in full-term placenta and is widely expressed in a variety of adult human tissues such as liver, skeletal, muscle, heart, kidney and pancreas (11-13). It is mainly synthesized and secreted into the ECM by a wide variety of cells. Given its pericellular location, TFPI-2 is thought to regulate the plasmin- and trypsin-mediated activation of matrix pro-metalloproteinases and play a significant role in the regulation of ECM degradation, which is an essential step for cell remodeling, as well as tumor cell invasion and metastasis (14).

Little is currently known regarding the role of protease inhibitors, particularly tissue factor pathway inhibitors, in HCC progression. Therefore, in this study, the role of TFPI-2 in HCC is examined.

\section{Materials and methods}

Tissue specimens. Human hepatocarcinoma tissues and tumor-adjacent normal hepatic tissues were obtained from HCC patients admitted to Shenzhen People's Hospital. The tissues were stored frozen at $-75^{\circ} \mathrm{C}$ until use.

In situ hybridization. Tumor specimens were fixed in formalin overnight and embedded in paraffin using standard procedures. Series sections $(4 \mu \mathrm{m})$ were deparaffinized with xylene, rehydrated in a graded series of ethanol and washed in PBS. Human TFPI-2 mRNA was detected using the in situ hybridization detection kit (Boster, Wuhan, China) according to the manufacturer's instructions. Briefly, the sections were hybridized in prehybridization buffer supplemented with $0.1 \mu \mathrm{g} / \mathrm{ml}$ digoxigenin-labeled, 1.2-kb antisense TFPI-2 probe overnight 
at $37^{\circ} \mathrm{C}$, incubated with biotinylated mouse anti-digoxigenin antibody (1:1000 dilution), and then incubated with biotinylated peroxidase. Staining was developed with DAB. Slides were counterstained with hematoxylin, dehydrated and mounted. The number of cells stained brown (indicating the presense of TFPI-2 mRNA) was assessed by light microscopy. The hybridization probe, replaced with phosphate-buffered saline (PBS) was used as a negative control. Mature placenta tissue, known to express large amounts of TFPI-2, was used as a positive control.

Immunohistochemistry. Tissue sections were prepared in the same manner as above. The expression of TFPI- 2 was then determined by incubation with a mouse polyclonal antibody against human TFPI-2 (Santa Cruz Biotechnology, Santa Cruz, CA, USA), horseradish peroxidase (HRP)-conjugated sheep anti-mouse $1 \mathrm{gG}$ secondary antibodies (Chinagen, Shenzhen, China). Detection was carried out using the non-biotin-labeled detection kit (Zhongshan Goldbridge, Beijing, China) according to the manufacturer's instructions. Staining was developed with $\mathrm{DAB}$, and slides were counterstained with hematoxylin, dehydrated and mounted. The primary antibody, replaced with PBS, was used as a negative control. Mature placenta tissue, known to express large amounts of TFPI-2, was used as a positive control.

Plasmid construct. A 0.7-kb fragment encoding TFPI-2 cDNA was amplified from normal liver tissue with the primers 5'-GCTTTCTCGGACGCCTTGC-3' and 5'-GAATACGACC CCAAGAAATGAGTGA-3'. PCR product was purified and cloned into the BamHI and XhoI sites of the pCDNA3.1expressing vector, donated by Dr Tiyuan Li (Central Laboratory, Shenzhen People's Hospital, China). The DNA sequence of the recombinant plasmid was confirmed via DNA sequencing.

Cell culture and transfection. Human hepatoma $\mathrm{HepG}_{2}$ cells were obtained from the Cancer Institute, Chinese Academy of Medical Sciences, and cultured in $6 \% \mathrm{CO}_{2}$ to $94 \%$ air and $96 \%$ humidity at $37^{\circ} \mathrm{C}$ in DMEM supplemented with $10 \%$ bovine calf serum (Hyclone, Logan, UT, USA), 1.0\% glutamine, $100 \mu \mathrm{g} / \mathrm{ml}$ streptomycin and $100 \mu \mathrm{g} / \mathrm{ml}$ penicillin. The recombinant constructs or pCDNA3.1 vector was transfected into $\mathrm{HepG}_{2}$ cells using Lipofectamine 2000 transfection reagent (Invitrogen, Carlsbad, CA, USA) according to the manufacturer's instructions. Selection of transfected cells with $0.8 \mathrm{mg} / \mathrm{ml} \mathrm{G} 418$ sulfate (Invitrogen) was initiated $48 \mathrm{~h}$ after transfection. After a 4-week selection, stable transfectants were expanded and used for the study. The $\mathrm{HepG}_{2}$ cells were divided into three groups: $\mathrm{HepG}_{2}$ parental cells $\left(\mathrm{HepG}_{2}-\mathrm{P}\right)$, $\mathrm{HepG}_{2}$ cells transfected by pCDNA 3.1 vector $\left(\mathrm{HepG}_{2}-\mathrm{V}\right)$ and $\mathrm{HepG}_{2}$ cells transfected by TFPI-2 construct (HepG $\mathrm{G}_{2}$-TFPI-2).

RT-PCR. Total RNA was isolated from $\mathrm{HepG}_{2}$ cells using TRIzol $^{\circledR}$ reagent (Invitrogen) according to a standard protocol. Using the 2-step (RT-PCR) kit (Takara, Dalian, China), cDNA was synthesized with RNA as the template. PCR amplification of human TFPI-2 and $\beta$-actin was performed with Taq Master Mix (Promega, Madison, WI, USA) with synthesized cDNA. The primers were synthesized by Shanghai Biotechnology (China) as follows: TFPI-2 5'-ATAGGATCCACATGGACCCGCTCGC-3' and 5'-GGCCTCGAGAAATTGCTTCTTCCGAATTTCC-3', amplicon 700 bp $\beta$-actin 5'-CTGGCACCACACCTTCTAC AATG-3' and 5'-AATGTCACGCACGATTTCCCGC-3'. The PCR conditions were: denaturation at $95^{\circ} \mathrm{C}$ for $30 \mathrm{sec}$, annealing at $52^{\circ} \mathrm{C}$ for $30 \mathrm{sec}$, and extension at $72^{\circ} \mathrm{C}$ for $40 \mathrm{sec}$ for 32 cycles. After electrophoresis of PCR products, the data were analyzed using Image Master Total Laboratory ID software (GE Healthcare, USA). The level of TFPI- 2 mRNA was calculated by the ratio of density of TFPI- 2 to $\beta$-actin.

Western blot analysis. $\mathrm{HepG}_{2}-\mathrm{P} \mathrm{HepG}_{2}$-TFPI-2 and $\mathrm{HepG}_{2}-\mathrm{V}$ cells were grown to $80-90 \%$ confluence in 6-well plates, after which the medium was replaced with serum-free medium and incubated for $24 \mathrm{~h}$. The cultures were then washed several times with PBS and the ECM was prepared as described by Rao et al (15). The ECM protein was supplemented with PMSF ( $1 \mathrm{mmol} / \mathrm{l})$ to inhibit the proteases. The samples were mixed with equal volume of $2 \mathrm{X}$ SDS sample buffer and boiled for $5 \mathrm{~min}$. Equal amounts of protein were resolved on $12 \%$ sodium dodecyl sulfate (SDS)-polyacrylamid gels, and then transfected onto polyvinylidene difluoride (PVDF) membrane (Millipore, Billerica, MA, USA). After blocking with $5 \%$ non-fat milk, the membranes were incubated, first with primary antibody at $4^{\circ} \mathrm{C}$ overnight and then with horseradish peroxidase (HRP)-conjugated sheep anti-mouse $1 \mathrm{gG}$ secondary antibody. After washing, the blots were developed with a super-enhanced chemiluminescence detection kit (Applygen Technologies, Beijing, China).

Cell proliferation assay. Cell proliferation was evaluated by MTT assay (Sigma, St. Louis, MO, USA) according to a procedure described previously (16). In brief, every $24 \mathrm{~h}$, for a total of 7 days, the cells from the three groups were harvested and $200 \mu \mathrm{l}$ cell suspension was added to each well in 96-well plates. A one-tenth volume of MTT solution (5 mg MTT/ml PBS) was added to each well and incubated for $2-4 \mathrm{~h}$ at $37^{\circ} \mathrm{C}$ until a purple precipitate was visible. The medium was then carefully removed, and precipitates were dissolved in $150 \mu \mathrm{l}$ DMSO. Growth rate was plotted as the percentage of viable cells in $\mathrm{HepG}_{2}-\mathrm{P}$ control (a value arbitrarily set at $100 \%$ ). Each experiment was repeated at least three times with each treatment given in duplicate or triplicate. Data were presented as an average of the results from individual experiments.

In vitro cell migration and invasion assay. Invasion and migration of the hepatocarcinoma cells in vitro was measured by the invasion of cells through Matrigel-coated or -uncoated transwell inserts according to a procedure described previously (17). Briefly, transwell inserts (Corning, Lowell, MA, USA) with $8-\mu \mathrm{m}$ pore size were coated (for invasion assay) with $50 \mu \mathrm{g}$ of matrigel matrix (BD Biosciences, San Jose, CA, USA) or uncoated (for the migration assay). Cells suspended in serum-free DMEM medium were seeded into upper chambers (100 $\mu \mathrm{l} /$ well) at a density of $3 \times 10^{5}$ cells $/ \mathrm{ml}$ (for the invasion assay) or $1 \times 10^{6}$ cells $/ \mathrm{ml}$ (for migration assay). The lower chambers were filled with DMEM and supplemented with $10 \%$ FBS. After $24 \mathrm{~h}$ of incubation, cells attached to the upper side of the filter were removed, and the filters were fixed and stained with hematoxylin and eosin. At this point, no difference was evident in the total number of cells (proliferation rate) among the groups in the serum-free medium. The number of 

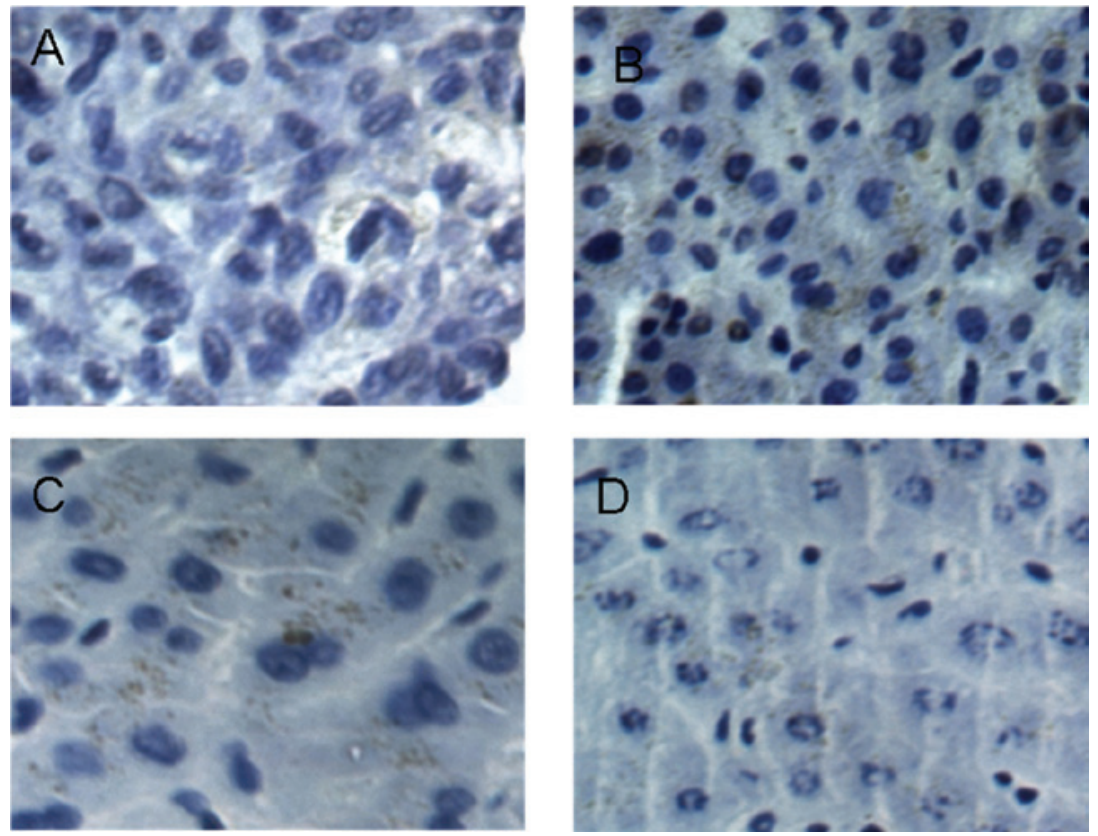

Figure 1. TFPI-2 expression in normal hepatic and hepatocarcinoma tissues. Expression of (A) TFPI-2 mRNA in hepatocarcinoma tissue and (B) tumor-adjacent normal hepatic tissue was examined by in situ hybridization with a digoxigenin-labeled TFPI-2 probe. Expression of (C) TFPI-2 protein in hepatocarcinoma tissue and (D) tumor-adjacent normal hepatic tissue was examined by immunohistochemical analyses with TFPI- 2 antibody. Magnification, $\mathrm{x} 400$.

cells that had migrated to the undersurface of the membrane was counted in five randomly-selected microscopic fields in each sample.

Statistical analysis. Data were presented as the mean \pm SD. Statistical analysis was performed with SPSS statistical software. The Student's two-tailed t-test was used to compare the difference between groups. $\mathrm{P}<0.05$ was considered to be statistically significant.

\section{Results}

TFPI-2 expression in normal hepatic and hepatocarcinoma tissues. In situ hybridization with TFPI-2 probe detected little or no TFPI-2 mRNA in hepatocarcinoma tissue sections, whereas a high level of TFPI-2 mRNA was detected in tumoradjacent normal hepatic tissue sections (Fig. 1A and B). The positive and negative controls confirmed the specificity of the hybridization liquid, replaced with PBS and used as a negative control, and the absence of a specific hybridization signal (data not shown).

Further immunohistochemical analysis confirmed that TFPI-2 protein was stained strongly positive in normal hepatic tissues but was weakly stained in hepatocarcinoma tissues (Fig. 1C and D). The TFPI-2 immunostaining scores for normal hepatic tissues and hepatocarcinoma tissues were $46.60 \pm 1.80$ and $22.54 \pm 1.22$, respectively $(\mathrm{P}<0.05)$. Taken together, these data indicate that the expression of TFPI- 2 was markedly reduced in hepatocarcinoma tissues.

TFPI-2 inhibits Hep $\mathrm{G}_{2}$ cell proliferation in vitro. To determine the functional role of TFPI-2 in $\mathrm{HCC}, \mathrm{HepG}_{2}$ cells were employed as a model. Using RT-PCR, the expression of TFPI-2 mRNA in $\mathrm{HepG}_{2}$ cells was undetectable (data not

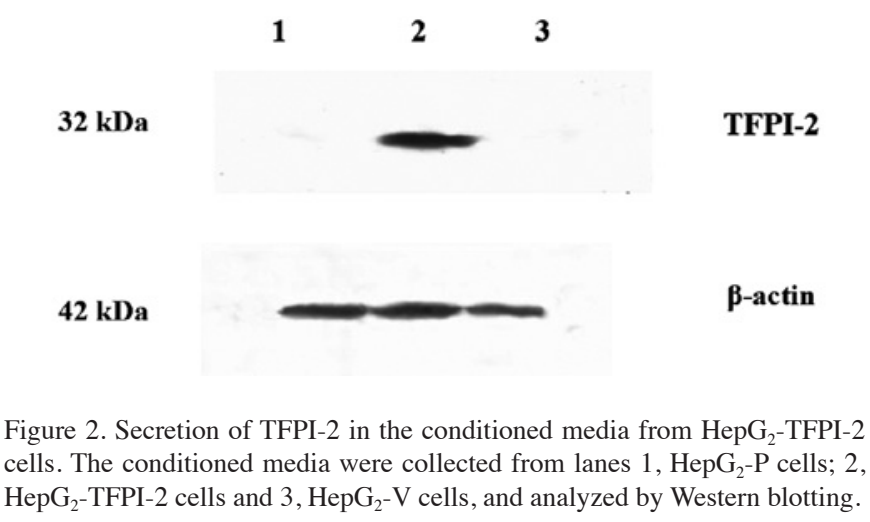

shown). Therefore, TFPI-2 was introduced into $\mathrm{HepG}_{2}$ cells by establishing a $\mathrm{HepG}_{2}$-TFPI-2 stable cell line. Using Western blotting, a high level of TFPI-2 protein was detected in the conditioned media of the $\mathrm{HepG}_{2}$-TFPI- 2 cells but not in that of $\mathrm{HepG}_{2}-\mathrm{V}$ or $\mathrm{HepG}_{2}-\mathrm{P}$ cells (Fig. 2). These results showed that TFPI-2 was successfully introduced into $\mathrm{HepG}_{2}$ cells.

The effect of TFPI-2 expression on the proliferation of $\mathrm{HepG}_{2}$ cells was then examined. Cell viability was determined by MTT assay for 7 days and cell proliferation was clearly inhibited on the fourth day in $\mathrm{HepG}_{2}$-TFPI-2 cells but not in the remaining two groups of cells (Fig. 3). These results suggested that TFPI-2 suppressed the growth of hepatocarcinoma cells.

TFPI-2 inhibits $\mathrm{HepG}_{2}$ cell invasion in vitro. The effect of TFPI-2 expression on the invasion of $\mathrm{HepG}_{2}$ cells was also examined. Based on the invasion and migration assays, the cells that passed through the membranes were counted (Table I). The results show that the number of cells passing through the membranes was significantly lower in the $\mathrm{HepG}_{2}$-TFPI-2 
Table I. In vitro invasion of different groups of HepG2 cells.

Membranes in matrigel invasion assay

\begin{tabular}{lcccccc}
\cline { 2 - 6 } Groups & 1 & 2 & 3 & 4 & 5 & $\chi \pm \mathrm{s}$ \\
\hline HepG $_{2}$-TFPI-2 & 45 & 53 & 39 & 48 & 41 & $49.3 \pm 5.9^{\mathrm{a}}$ \\
$\mathrm{HepG}_{2}$-V & 91 & 93 & 80 & 79 & 88 & $86.2 \pm 6.4$ \\
$\mathrm{HepG}_{2}$-P & 90 & 86 & 93 & 81 & 89 & $87.8 \pm 4.5$
\end{tabular}

${ }^{\mathrm{a}} \mathrm{P}<0.05$ vs. $\mathrm{HepG}_{2}-\mathrm{V}$ or $\mathrm{HepG}_{2}-\mathrm{P}$.

\begin{tabular}{lcccccc}
\hline & \multicolumn{5}{c}{ Membranes in migration assay } \\
\cline { 2 - 7 } Groups & 1 & 2 & 3 & 4 & 5 & $\chi \pm \mathrm{S}$ \\
\hline $\mathrm{HepG}_{2}$-TFPI-2 & 142 & 132 & 139 & 152 & 137 & $140.4 \pm 7.4$ \\
$\mathrm{HepG}_{2}$-V & 134 & 148 & 160 & 140 & 147 & $145.8 \pm 9.8$ \\
$\mathrm{HepG}_{2}$-P & 150 & 166 & 159 & 142 & 147 & $152.8 \pm 9.6$ \\
\hline
\end{tabular}

group than in the remaining two groups $(\mathrm{P}<0.05)$, indicating that TFPI-2 suppresses the invasive potential of hepatocarcinoma cells, whereas no significant difference in migration ability was observed in the three groups (Table I).

\section{Discussion}

TFPI-2 is a serine proteinase inhibitor, which is frequently downregulated in malignant tumors (18). Previous studies have demonstrated that silencing of TFPI-2 by either histone deacetylation (19) or promoter hypermethylation contributes to its inactivation and tumor progression in a variety of cancers including glioma (18), choricarcinoma (20), pancreatic carcinoma (17), lung carcinoma (21), breast cancer (22), melanoma (23) and hepatocarcinoma (24). In addition, the aberrant splicing form of TFPI-2 was detected during cancer progression (25), which represents an untranslated form providing another mechanism by which TFPI-2 is downregulated in tumor cells.

In this study, the expression and role of TFPI-2 in HCC were investigated. First, in situ hybridization and immunohistochemistry methods were applied to evaluate the expression of TFPI- 2 mRNA and protein in hepatocarcinoma and tumoradjacent normal hepatic tissues. Consistent with previous studies, the results showed that TFPI-2 expression at the mRNA and protein levels was low in hepatocarcinoma tissues compared to adjacent normal hepatic tissues. These results indicated that a decreased expression of TFPI- 2 is involved in HCC.

To determine the mechanism by which TFPI- 2 loss contributes to $\mathrm{HCC}, \mathrm{HepG}_{2}$ cells were employed as a model. The results demonstrate that reconstitution of TFPI-2 into $\mathrm{HepG}_{2}$ cells inhibited the proliferation and invasion of $\mathrm{HepG}_{2}$ cells. Although the details of TFPI-2-mediated growth suppression are unknown, a previous study suggested that TFPI-2 induces apoptosis in glioma cells (26). Further studies are required to examine whether TFPI-2 promotes the apoptosis of $\mathrm{HepG}_{2}$ cells. In agreement with previous reports asserting that the overexpression of TFPI-2 reduced the invasion of cancer

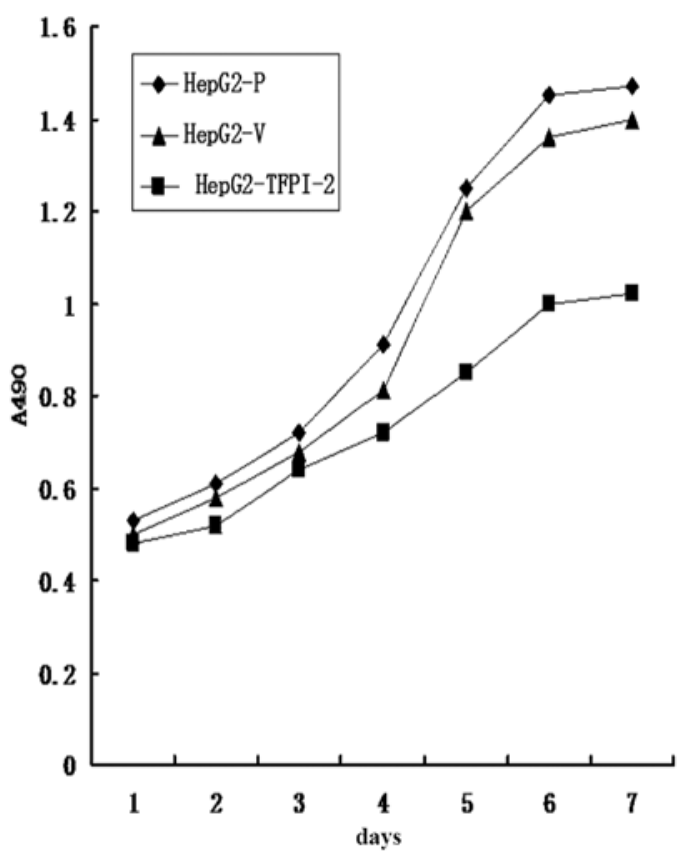

Figure 3. The growth curve of different groups of $\mathrm{HepG}_{2}$ cells $\left(\mathrm{HepG}_{2}-\mathrm{P}\right.$, $\mathrm{HepG}_{2}-\mathrm{V}$ and $\mathrm{HepG}_{2}-\mathrm{TFPI}-2$ ).

cell lines derived from melanoma (27), prostate cancer (28), choriocarcinoma (29), glioblastoma (30) or meningiomas (31), our results showed that the restoration of TFPI- 2 was associated with a 2 -fold decrease in the invasive ability of $\mathrm{HepG}_{2}$ cells. TFPI-2 is thought to play a pivotal role in the regulation of plasmin-mediated ECM proteolysis during tumor invasion and metastasis (14). TFPI-2 inhibits the release of plasmin and trypsin, preventing activation of pro-matrix metalloproteinase (MMP)-1 and pro-MMP-3, leading to diminished ECM degradation and decreased invasion of HT-1080 fibrosarcoma cell lines $(32,33)$. In addition, TFPI-2 is capable of inhibiting MMP-2 activation in the HT-1080 fibrosarcoma cell line (34) and directly inhibits MMP-1, MMP-13, MMP-2 and MMP-9 
in experimental models (35). Thus, we postulate that additional mechanisms responsible for TFPI-2 inhibit HCC invasion and metastasis by modulating the activity of MMPs.

In conclusion, we reported that TFPI-2 expression is lost in HCC. The results of our in vitro studies confirm that restoration of TFPI-2 caused decreased proliferative and invasive behaviors of $\mathrm{HepG}_{2}$ cells. Taken together, these data suggest that inactivation of TFPI-2 contributes to malignant behavior in hepatocarcinoma, suggesting the potential role of TFPI-2 as a tumor suppressor in HCC. However, additional in vivo studies may be useful to determine whether restoration of TFPI-2 in hepatocarcinoma cells is a novel therapeutic approach for HCC.

\section{Acknowledgements}

We gratefully acknowledge all the members of the laboratory in the People's Hospital of Shenzhen, China for their technical assistance and advice throughout this study. Special thanks is extended to Dr Tiyuan Li for providing the PCDNA 3.1 vector and technical assistance. This study was supported by grants from the Bureau of Science and Technology in Shenzhen City and the Department of Health in Guangdong Province, China.

\section{References}

1. Okuda K: Hepatocellular carcinoma: recent progress. Hepatology 15: 948-963, 1992.

2. Enzan H, Himeno H, Iwamura S, et al: Alpha-smooth muscle actin-positive perisinusoidal stromal cells in human hepatocellular carcinoma. Hepatology 19: 895-903, 1994.

3. Faouzi S, Le Bail B, Neaud V, et al: Myofibroblasts are responsible for collagen synthesis in the stroma of human hepatocellular carcinoma: an in vivo and in vitro study. J Hepatol 30: 275-284, 1999.

4. Terada T, Makimoto K, Terayama N, Suzuki Y and Nakanuma Y: Alpha-smooth muscle actin-positive stromal cells in cholangiocarcinomas, hepatocellular carcinomas and metastatic liver carcinomas. J Hepatol 24: 706-712, 1996.

5. Egeblad M and Werb Z: New functions for the matrix metalloproteinases in cancer progression. Nat Rev Cancer 2: 161-174, 2002.

6. Stamenkovic I: Extracellular matrix remodelling: the role of matrix metalloproteinases. J Pathol 200: 448-464, 2003.

7. Vihinen P and Kahari VM: Matrix metalloproteinases in cancer: prognostic markers and therapeutic targets. Int J Cancer 99: $157-166,2002$

8. Westermarck J and Kahari VM: Regulation of matrix metalloproteinase expression in tumor invasion. Faseb J 13: 781-792, 1999.

9. Noel A, Jost M and Maquoi E: Matrix metalloproteinases at cancer tumor-host interface. Semin Cell Dev Biol 19: 52-60, 2008.

10. Butzow R, Huhtala ML, Bohn H, Virtanen I and Seppala M: Purification and characterization of placental protein 5. Biochem Biophys Res Commun 150: 483-490, 1988

11. Sprecher CA, Kisiel W, Mathewes S and Foster DC: Molecular cloning, expression, and partial characterization of a second human tissue-factor-pathway inhibitor. Proc Natl Acad Sci USA 91: 3353-3357, 1994.

12. Miyagi Y, Yasumitsu H, Eki T, et al: Assignment of the human PP5/TFPI-2 gene to 7q22 by FISH and PCR-based human/rodent cell hybrid mapping panel analysis. Genomics 35: 267-268, 1996.

13. Miyagi Y, Koshikawa N, Yasumitsu H, et al: cDNA cloning and mRNA expression of a serine proteinase inhibitor secreted by cancer cells: identification as placental protein 5 and tissue factor pathway inhibitor-2. J Biochem 116: 939-942, 1994.

14. Chand HS, Du X, Ma D, et al: The effect of human tissue factor pathway inhibitor-2 on the growth and metastasis of fibrosarcoma tumors in athymic mice. Blood 103: 1069-1077, 2004.

15. Rao CN, Reddy P, Liu Y, et al: Extracellular matrix-associated serine protease inhibitors $(\mathrm{Mr} 33,000,31,000$, and 27,000) are single-gene products with differential glycosylation: cDNA cloning of the 33-kDa inhibitor reveals its identity to tissue factor pathway inhibitor-2. Arch Biochem Biophys 335: 82-92, 1996.
16. Tang Z, Geng G, Huang Q, et al: Expression of tissue factor pathway inhibitor 2 in human pancreatic carcinoma and its effect on tumor growth, invasion, and migration in vitro and in vivo. J Surg Res 167: 62-69, 2009.

17. Sato N, Parker AR, Fukushima N, et al: Epigenetic inactivation of TFPI-2 as a common mechanism associated with growth and invasion of pancreatic ductal adenocarcinoma. Oncogene 24: 850-858, 2005.

18. Konduri SD, Srivenugopal KS, Yanamandra N, et al: Promoter methylation and silencing of the tissue factor pathway inhibitor-2 (TFPI-2), a gene encoding an inhibitor of matrix metalloproteinases in human glioma cells. Oncogene 22: 4509-4516, 2003.

19. Pulukuri SM, Gorantla B and Rao JS: Inhibition of histone deacetylase activity promotes invasion of human cancer cells through activation of urokinase plasminogen activator. J Biol Chem 282: 35594-35603, 2007.

20. Hube F, Reverdiau P, Iochmann S, Rollin J, Cherpi-Antar C and Gruel Y: Transcriptional silencing of the TFPI-2 gene by promoter hypermethylation in choriocarcinoma cells. Biol Chem 384: 1029-1034, 2003.

21. Rollin J, Iochmann S, Blechet C, et al: Expression and methylation status of tissue factor pathway inhibitor-2 gene in nonsmall-cell lung cancer. Br J Cancer 92: 775-783, 2005.

22. Guo H, Lin Y, Zhang H, et al: Tissue factor pathway inhibitor-2 was repressed by $\mathrm{CpG}$ hypermethylation through inhibition of KLF6 binding in highly invasive breast cancer cells. BMC Mol Biol 8: 110, 2007.

23. Nobeyama Y, Okochi-Takada E, Furuta J, et al: Silencing of tissue factor pathway inhibitor-2 gene in malignant melanomas. Int J Cancer 121: 301-307, 2007.

24. Wong CM, Ng YL, Lee JM, et al: Tissue factor pathway inhibitor- 2 as a frequently silenced tumor suppressor gene in hepatocellular carcinoma. Hepatology 45: 1129-1138, 2007.

25. Kempaiah P, Chand HS and Kisiel W: Identification of a human TFPI-2 splice variant that is upregulated in human tumor tissues. Mol Cancer 6: 20, 2007

26. Tasiou A, Konduri SD, Yanamandra N, et al: A novel role of tissue factor pathway inhibitor-2 in apoptosis of malignant human gliomas. Int J Oncol 19: 591-597, 2001.

27. Konduri SD, Tasiou A, Chandrasekar N, Nicolson GL and Rao JS: Role of tissue factor pathway inhibitor-2 (TFPI-2) in amelanotic melanoma (C-32) invasion. Clin Exp Metastasis 18: 303-308, 2000

28. Konduri SD, Tasiou A, Chandrasekar N and Rao JS: Overexpression of tissue factor pathway inhibitor-2 (TFPI-2), decreases the invasiveness of prostate cancer cells in vitro. Int J Oncol 18: 127-131, 2001 .

29. Jin M, Udagawa K, Miyagi E, et al: Expression of serine proteinase inhibitor PP5/TFPI-2/MSPI decreases the invasive potential of human choriocarcinoma cells in vitro and in vivo. Gynecol Oncol 83: 325-333, 2001.

30. Yanamandra N, Kondraganti S, Gondi CS, et al: Recombinant adeno-associated virus (rAAV) expressing TFPI-2 inhibits invasion, angiogenesis and tumor growth in a human glioblastoma cell line. Int J Cancer 115: 998-1005, 2005.

31. Kondraganti S, Gondi CS, Gujrati M, et al: Restoration of tissue factor pathway inhibitor inhibits invasion and tumor growth in vitro and in vivo in a malignant meningioma cell line. Int J Oncol 29: 25-32, 2006.

32. Rao CN, Cook B, Liu Y, et al: HT-1080 fibrosarcoma cell matrix degradation and invasion are inhibited by the matrix-associated serine protease inhibitor TFPI-2/33 kDa MSPI. Int J Cancer 76: 749-756, 1998.

33. Rao CN, Mohanam S, Puppala A and Rao JS: Regulation of ProMMP- 1 and ProMMP-3 activation by tissue factor pathway inhibitor-2/matrix-associated serine protease inhibitor. Biochem Biophys Res Commun 255: 94-98, 1999.

34. Izumi $\mathrm{H}$, Takahashi $\mathrm{C}$, Oh $\mathrm{J}$ and Noda $\mathrm{M}$ : Tissue factor pathway inhibitor-2 suppresses the production of active matrix metalloproteinase- 2 and is down-regulated in cells harboring activated ras oncogenes. FEBS Lett 481: 31-36, 2000.

35. Herman MP, Sukhova GK, Kisiel W, et al: Tissue factor pathway inhibitor-2 is a novel inhibitor of matrix metalloproteinases with implications for atherosclerosis. J Clin Invest 107: 1117-1126, 2001. 